\title{
Neuroglial interactions underpinning myelin plasticity
}

Omar de Faria Jr*, E.A. Claudia Pama*, Kimberley Evans, Aryna Luzhynskaya \& Ragnhildur T Káradóttir ${ }^{\dagger}$

Wellcome Trust-Medical Research Council Cambridge Stem Cell Institute \& Department of Veterinary Medicine, University of Cambridge, Cambridge, United Kingdom

* equal contribution

${ }^{\dagger}$ Send correspondence to: Ragnhildur Thóra Káradóttir, Wellcome Trust - Medical Research Council Stem Cell Institute, University of Cambridge, Tennis Court Road, Cambridge, CB2 1QR, UK. E-mail: rk385@,cam.ac.uk 


\begin{abstract}
:
The CNS is extremely responsive to an ever-changing environment. Studies of neural circuit plasticity focus almost exclusively on functional and structural changes of neuronal synapses. In recent years, however, myelin plasticity has emerged as a potential modulator of neuronal networks. Myelination of previously unmyelinated axons, and changes in the structure of myelin on already-myelinated axons (like changes in internode number and length or myelin thickness or geometry of the nodal area) can in theory have significant effects on the function of neuronal networks. Here we review the current evidence for myelin changes occurring in the adult CNS, highlight some potential underlying mechanisms of how neuronal activity may regulate myelin changes, and explore the similarities between neuronal and myelin plasticity.
\end{abstract}

\title{
Keywords:
}

Myelination

Myelin

Oligodendrocyte

Neuronal activity

Neurotransmitters 


\section{Introduction}

Myelin, produced by oligodendrocytes in the CNS, is essential for rapid information transmission and synchronisation of axonal inputs among billions of neurons. Myelin plasticity is now increasingly invoked as a fundamental mechanism for learning (see reviews in this issue). Observations on human myelin formation in the early 1900s (Flechsig, 1901; Kaes, 1907; Yakovlev and Lecours, 1967) had identified that myelination continues into late adult life and studies in the end of the last century showed that new myelinating oligodendrocytes are formed in adult animals (Reyners et al., 1986; Rivers et al., 2008). Yet it was not until diffusion tensor imaging (DTI) was used in humans, highlighting structural changes in the white matter following training (Bengtsson et al., 2005; Carreiras et al., 2009; Scholz et al., 2009), that a potential role of myelin in learning was hypothesized. Although it is still unclear whether white matter structural changes indicate myelin remodelling (Walhovd et al., 2014; Zatorre et al., 2012), environmental enrichment (and social isolation) alters myelination in mice (Liu et al., 2012; Makinodan et al., 2012), the acquisition of new motor tasks (McKenzie et al., 2014; Sampaio-Baptista et al., 2013) increases myelination and these changes seem to be required for learning (McKenzie et al., 2014). These finding thus argue in favour of the interpretation that increases in FA detected by DTI are indeed due to myelination changes. Thus, myelin - previously considered static - might in fact be plastic and responsive to changes in neuronal activity. In this perspective, we review the evidence for myelin plasticity and postulate on the potential mechanisms by which neuronal activity might regulate it.

\section{Developmental myelination and white matter plasticity}

Early histological studies on post-mortem human brains identified four important aspects of human myelination. First, myelination is a protracted process that is ongoing far into adulthood, well into the sixth decade (Figure 1) (Kaes, 1907; Yakovlev and Lecours, 1967). Second, myelination of different brain areas follows a clear temporal sequence, which is similar across species (Gibson, 1970). In this context, Flechsig, in 1901, after a lifetime of post-mortem studies, put forward a fundamental law for myelination - 'the myelinogenetic law' - which states "that the myelinization of nerve fibers in the developing brain follows a definite chronologic sequence such that those fibers belonging to particular functional systems mature at the same time" (Flechsig, 1901; Flechsig, 1920). This law did not go unchallenged, but careful behavioural and histological studies of kittens supported the law as the achievement of behavioural milestones consistently coincided with the relevant tracts being myelinated (Tilney and Casamajor, 1924). Flechsig further classified 3 temporal stages of myelination depending upon the timing of myelination (1) early myelination 


\section{PLEASE INSERT FIGURE 1 HERE}

(foetus, at birth); (2) intermediate (childhood) and (3) late myelination (starting around adolescent to adult) (Flechsig, 1901). It is now well accepted that projection fibers are myelinated before the commissural fibers and that the last fiber tracts to become myelinated are the association fibers, which continue to myelinate far into adulthood (Gibson, 1970; Tilney and Casamajor, 1924; Yakovlev and Lecours, 1967). The third aspect identified in these early studies is that there are different myelination cycles (the time from the onset of myelination to its completion) which govern how different areas myelinate: (1) monophasic myelination cycle (fast completion from onset of myelination), (2) multi-phasic myelination cycle (where there is an early onset but then a delay prior to completion of the cycle), or (3) slow continuous protracted myelination cycle. The two last cycles tend to be detected in brain areas that myelinate over the time-course of human development (Flechsig, 1901; Miller et al., 2012; Yakovlev and Lecours, 1967). Lastly, myelination within each area/tract follows a stereotypical pattern, where axons of larger diameter are myelinated first, followed by myelination of smaller diameter axons (Matthews and Duncan, 1971; Remahl and Hildebrand, 1982). How these different onsets and cycles of myelination are regulated is still unclear, but it is conceivable that for the CNS there may be a number of different mechanisms regulating myelination, each for a different brain area and/or myelination cycle.

Novel imaging methods have validated most of the early histological observations that myelination proceeds from deep to superficial brain regions (Kinney et al., 1988; Yakovlev and Lecours, 1967), with the association fibers being myelinated last. For example the uncinate fasciculus, which connects the limbic regions in the temporal lobe to the frontal lobe, is one of the last tracts to become myelinated and shows the greatest individual variability in myelination, which has been associated with differences in behaviour (Olson et al., 2015). In line with this, areas that myelinate early in development, such as the corticospinal tract, show less individual variability than areas that myelinate late, such as the association fibers (Bürgel et al., 2006). This may suggest that areas that are myelinated before or shortly after birth have an intrinsic less-changeable myelination programme, but areas that myelinate late, with larger individual variance, are myelinated by a programme that can adjust to changes in the environment of the individual.

Several factors in the environment seem to be able to modulate white matter (Bergmann and Frisen, 2013; Fields, 2008; Liu et al., 2012; Makinodan et al., 2012; Etxeberria et al., 2016) and would therefore alter the conduction speed of neuronal signalling (Pajevic et al., 2014; Ford et al., 2015; Etxeberria et al., 2016; Arancibia-Cárcamo et al., 2017). There is growing 
evidence indicating that learning a new skill might increase white matter volume, as well as findings demonstrating that myelination correlates with the learning observed (Hofstetter et al., 2013; Lakhani et al., 2016; Long and Corfas, 2014). For example, piano practising during childhood has been associated with an increase in white matter volume and changes in the structure of various fibre tracts in adulthood, as measured with DTI (Bengtsson et al., 2005). In addition, working memory training correlates with an increase in fractional anisotropy (FA) in the relevant white matter region, suggesting that this type of learning leads to increased myelination (Takeuchi et al., 2010). Similarly, learning a complex visuomotor skill such as juggling (Scholz et al., 2009) increases white matter structural changes in the intraparietal sulcus. White matter changes have been further observed during various types of skill learning such as reading, where white matter increases in the corpus callosum are linked to interhemispheric connectivity that coincides with learning this skill (Carreiras et al., 2009).

The question arises as to what extent modifications in white matter structure identified in human imaging studies are indicative of myelin changes. Although many advances in quantifying myelination have been made in the field of imaging, myelin-specific measures that utilise different acquisition methods (or pulse sequences) that estimate myelin water fraction - such as magnetization transfer (MT; see Whitaker et al., 2016, Zhang et al 2015) and mcDESPOT (Deoni et al., 2011, Zhang et al. 2015) - are being developed and optimised. These methods argue in favour of the interpretation that FA changes as measured with DTI are due to differences in myelination. Whether macro-scale white matter findings correspond to micro-scale changes in myelin can only be directly addressed by using animal models. Fractional anisotropy and MBP immunoreactivity are also elevated in rodent subcortical white matter regions following motor and cognitive training (Blumenfeld-Katzir et al., 2011; Sampaio-Baptista et al., 2013). Furthermore, social isolation or sensory deprivation in rodents decreases myelin thickness and reduces the number of myelinated axons in vivo (Barrera et al., 2013; Gyllensten and Malmfors, 1963; Makinodan et al., 2012; Liu et al., 2012), whereas an increase of sensory input potentiates myelin formation (Tauber et al., 1980) and learning new motor tasks increases new myelinating oligodendrocytes (McKenzie et al., 2014). Thus, supporting that myelination changes are a component in changes in FA and oligodendrocyte lineage cells can 'respond' to changes, in neuronal activity, associated with learning (see BOX 1 and Figure 2 for different forms in which myelin might change). 


\section{BOX 1:}

\section{Different forms of myelin plasticity}

We can envisage a number of non-exclusive forms of myelin plasticity (see Figure 2).

\section{Unmyelinated axons become myelinated}

One possibility for activity-dependent myelination would be new myelin formation on unmyelinated axons. A substantial proportion of axons remain unmyelinated in many myelinated tracts of the adult CNS (Sturrock, 1980). This is particularly apparent for small diameter axons $(0.4-0.8 \mu \mathrm{m})$, which are comparable in calibre, but can be either myelinated or unmyelinated (Hildebrand et al., 1993). This number of unmyelinated axons in the adult CNS allows, therefore, for a large dynamic range in plasticity, whereby unmyelinated axons become myelinated potentially as a function of their activity. In fact, an abundant and widespread population of adult OPCs continuously differentiate into mature oligodendrocytes that are capable of myelinating these tracts (Dawson et al., 2003) and both experience and artificial stimulation of neuronal circuits induce oligodendrogenesis and de novo myelination (Simon et al., 2011; McKenzie et al., 2014; Gibson et al., 2014). Myelination of previously unmyelinated axons, presumably by an activity-dependent form of myelination, can provide a potential, and perhaps effective, form of myelin plasticity.

\section{New internodes are being formed}

Another possible manifestation of activity-dependent myelination involves the formation of new internodes to unmyelinated segments of axons. Myelinated cortical neurons from the same area, similar in size and axonal diameter are not equally myelinated along the entire length of their axons, as traditionally thought (Deschenes and Landry, 1980; Remahl and Hildebrand, 1982; Tomassy et al., 2014). In fact, long unmyelinated axon segments randomly intersperse between myelinated portions of individual axons suggesting that, in addition to unmyelinated axons, unmyelinated segments of myelinated axons are a substrate for myelination. In addition, it is possible that many axons regarded as unmyelinated in white matter tracts might in fact correspond to partially myelinated axons, containing long unmyelinated regions. 
Insertion of new internodes can alter action potential conduction velocity and thus change the timing of signal propagation, affecting the dynamics of neural circuitry processing. Changes of internodal length and number were shown to be an important mechanism underlying coincidence detection in the auditory system, where distinct patterns of myelination in cochlear neuron collateral branches correlate with differential conduction velocity, tuned to allow for temporal summation of inputs arising from both ears (Seidl and Rubel, 2016; Seidl et al., 2014). Differential myelination of olivocerebellar and thalamocortical fibres was also shown to individually regulate conduction velocity of axons within these tracts and, in addition, to be required for synchronized activity of populations of cerebellar Purkinje cells and cortical neurons onto which these fibres synapse (Lang and Rosenbluth, 2003; Salami et al., 2003). Therefore, de novo myelination may provide a novel mechanism for neural plasticity, in which activity-dependent changes in myelin profiles fine-tune conduction delays of individual fibres in such a way that circuit activity is optimized.

\section{Modulation of existing myelin}

In contrast to de novo myelination, existing myelin could also be changed in the process of myelin plasticity. In humans, remodelling of myelin has been shown to occur more frequently than the myelination of unmyelinated axons (Yeung et al., 2014) and this is possibly an important component of myelin plasticity. In fact, myelinating oligodendrocytes are able to sense neurotransmitters (Micu et al., 2016) released by axonal activity, and to increase myelin thickness in response to neuronal activity (Gibson et al., 2014); conversely, social deprivation and reduced neuronal firing results in reduction of myelin g-ratio (Makinodan et al., 2012; Liu et al., 2012) Thus, it is not surprising that the relation between axonal diameter, myelin thickness and internode length is largely variable in the adult CNS, possibly reflecting myelin remodelling of mature circuits. In particular, the variability in internode length may arise from the formation of new myelin, as adult born oligodendrocytes generate internodes that are shorter, or in some cases much longer, than internodes generated during development (Young et al., 2013), but can also result from changes in neuronal activity and neurotransmitter signalling, since inhibitory GABAergic signalling regulates internode length on glutamatergic axons (Hamilton et al., 2017). Whether these changes are bidirectional, that is, once myelin internodes are formed they can decrease in length and/or thickness, remains to be demonstrated. But it is evident that an increase in thickness on the existing myelin seems to occur (Gibson et al., 2014) and therefore changes in myelin structure is another potential form of myelin plasticity in the adult CNS. 


\section{Nodes of Ranvier}

Finally, a fourth way in which myelin plasticity could occur is through changes in the nodes of Ranvier. It has been suggested that axons might be able to modify the nodal length, both in health and in disease, to regulate speed of conduction and the delay of signal propagation (Huff et al., 2011; Trigo and Smith, 2015). A recent study has provided evidence supporting this concept and implying that changes in the geometry of the nodes of Ranvier may function as a form of myelin plasticity (Arancibia-Carcamo et al., 2017). In this study, measurements of the nodal length in the optic nerve and cerebral cortex revealed a large fold-range in length variability -4.4 and 8.7 -fold, respectively. A variation of this magnitude may be of functional relevance, as computational modelling of axonal conduction velocity estimated a corresponding effect of $20 \%$ in the speed of impulse propagation, similar to the range of speed variation resulting from changes in internode length and myelin thickness. Since the membrane area needed for changes in conduction speed is much smaller for nodes of Ranvier, this mechanism could represent a faster and energy-efficient form of myelin plasticity (Arancibia-Carcamo et al., 2017).

\section{Experience driven myelination}

Whether these experience-driven changes in myelin are necessary for learning or simply reflect adjustments to new circuit requirements, happening only after learning has occurred, are the subjects of current research.

Oligodendrocyte precursor cells (OPCs), which differentiate into myelinating oligodendrocytes during development, are equally distributed throughout the adult brain and are around 5\% of all the cells in the adult CNS (Dawson et al., 2003). Although their role in the adult brain is not fully understood, a significant proportion of oligodendrocytes continues to be produced in the adult CNS (Young et al., 2013), and BrdU incorporation studies indicate that the size of the oligodendrocyte population increases during the adult life (Dawson et al., 2003), thus indicating that adult OPCs both replenish and continuously produce new myelinating oligodendrocytes. OPC/oligodendrocyte dynamics appears to be regulated by experience, as changes in the environment modulate OPC proliferation and physical activity stimulates OPC cell cycle exit and differentiation (Okuda et al., 2009; Simon et al., 2011). In addition, acquiring a new motor skill increases OPC proliferation and oligodendrocyte generation and newborn oligodendrocytes are detected within only 2.5 hours of initiating 
motor training (McKenzie et al., 2014; Xiao et al., 2016). Rapid induction of oligodendrocyte production likely results from direct differentiation of G1-phase paused OPCs and, consistently with this hypothesis, OPC quantities are transiently reduced following training, before a new burst of proliferation replenishes OPC numbers to its homeostatic levels (Hughes et al., 2013; Xiao et al., 2016).

Remarkably, while learning a new sensory-motor skill directly increases the number of newly myelinating oligodendrocytes, blocking of adult oligodendrocyte production inhibits motor learning, suggesting that myelin changes are needed for correct execution of the task or for learning to occur (McKenzie et al., 2014). New findings regarding the timing of these learning-induced changes seem to further support the idea that myelin and oligodendrocytes may be necessary components in learning. Myelin basic protein (MBP) translation has been reported to happen within minutes to hours of neuronal activity (Südhof, 2013; Wake et al., 2011) and recently Xiao et al (2016) identified the existence of responsive pre-myelinating oligodendrocytes (identified with the Ennp6 marker) which lead to oligodendrocyte production astoundingly early (within 2 hours) in motor learning. These surprisingly fast dynamics of oligodendrocyte production is comparable to rapid changes in number and size of dendritic spines that occurs during synaptic plasticity (Xu et al., 2009). Since myelin sheath formation occurs during a restricted, short period following oligodendrocyte formation (Czopka et al., 2013), myelin plasticity may indeed function in close partnership with synaptic plasticity, as an additional mechanism underlying learning and memory. In addition, it seems that not only does myelination change as a reaction to the environment, but that these changes also have crucial long term behavioural and cognitive consequences, as demonstrated by studies investigating the correlates of early social isolation (associated with cognitive and behavioural symptoms) and white matter changes in adulthood (Liu et al., 2012; Makinodan et al., 2012). Taken together, these results suggest that experience regulates myelination and that myelin is plastic - yet how these changes occur is unclear. Which mechanisms underlie myelin plasticity in the adult CNS?

\section{PLEASE INSERT FIGURE 2 HERE}

\section{Neuronal activity-regulated myelination}

Many studies indicate that myelination of several white matter tracts requires neuronal firing and that neuronal activity is a mechanism employed by experience to drive myelin plasticity. Similarly to experience, neuronal activity regulates OPC/oligodendrocyte cell number, as block of activity in vivo with TTX alters OPC proliferation (Barres and Raff, 1993; Gautier et al., 2015) and electrical stimulation of adult motor cortical neurons increases OPC 
proliferation and oligodendrocyte differentiation along the corticospinal tract and the subcortical white matter (Gibson et al., 2014; Li et al., 2010). Consistent with these changes in oligodendrocyte number dynamics, block of synaptic transmission impairs myelination (and remyelination) in vivo (Hines et al., 2015; Mensch et al., 2015; Wake et al., 2011; Gautier et al., 2015; Etxeberria et al., 2016), and TTX eye injection diminishes the myelination in the optic nerve (Demerens et al., 1996), whereas optogenetic stimulation of the premotor cortex results in increased myelin thickness and improved motor function in vivo (Gibson et al., 2014).

These results suggest that neuronal activity is an important regulator for myelin changes in the CNS. However, myelination can also occur in the absence of neuronal activity (Bechler et al., 2015; Colello et al., 1995; Colello and Pott, 1997; Lee et al., 2013; Rosenberg et al., 2008; Shrager and Novakovic, 1995). These results potentially indicate that neuronal activity is a modulator of myelination rather than a regulator, particularly as when vesicular release of glutamate is prevented in the optic nerve the same number of axons are myelinated but with shorter internodes (Etxeberria et al., 2016). Another potential explanation for these contrasting observations, is provided by the findings that two distinct modes of myelination exist: one that is independent of axonal activity, and one that depends on active neurons releasing glutamate that activate glutamate receptors on oligodendrocytes lineage cells (Koudelka et al., 2016; Lundgaard et al., 2013). When levels of the growth factors Neuregulin (NRG1) or Brain-derived Neurotrophic Factor (BDNF) are elevated, presumably released from active neurons (Balkowiec and Katz 2002; Ozaki et al., 2004), OPCs switch to myelinating via an activity-dependent mechanism, which is a faster mode of myelination than the activity-independent myelination (Lundgaard et al., 2013; BOX 2 \& Figure 3). In line with this, developmental myelination is slowed (Kougioumtzidou et al., 2017; Saab et al., 2016) but relatively unaffected by knock-out of NMDARs (De Biase et al., 2011; Saab et al., 2016) or AMPARs (Kougioumtzidou et al., 2017) in oligodendrocyte lineage cells, presumably because developmental myelination can still occur by the activity-independent mode of myelination. Similarly, deleting ErbB3, a receptor for NRG1, in oligodendrocyte lineage cells has little effect on developmental myelination but disrupts experience-dependent myelination (Makinodan et al., 2012). Therefore, to address the mechanisms and the role of activity dependent myelination, the timing of the manipulations may be critical, similar to development of neuronal circuits where a critical period exists and depending on when mechanisms of activity are manipulated lead to different effects on the circuit (Turrigiano, 2011; see also BOX 2). The relative importance of each mode of myelination is still unclear. But, the mode of myelination which is employed seems to be axon specific, at least in embryonic development (Koudelka et al., 2016). It is possible that CNS regions myelinated 
before or shortly after birth are myelinated primarily by an innate myelination programme, independent of neuronal activity. In contrast, tracts or circuits which depend on experience for correct development, and areas that myelinate late and show a larger individual variance, are primarily myelinated by a programme that can adjust to changes in the environment of the individual and is dependent on neuronal activity. Similarities with these two modes of myelination can be drawn from circuit development, where both intrinsic programme, independent of neuronal firing rate and a programme dependent of neuronal activity are employed, and the dependence on each is developmentally regulated (Turrigano, 2011; Zhang and Poo 2001; see also BOX 2). Conceivably activity-dependent myelination might have evolved in order to speed up, as it is a faster mode of myelination (Lundgaard et al., 2013), and target myelination to occur on 'correctly' firing active axons during periods of developmental plasticity or learning, and thus may be important to fine-tune neuronal circuits that are shaped by activity. 


\section{BOX 2:}

\section{Developmental myelination or myelin plasticity?}

Myelination in humans extends well into adulthood, with certain brain areas being robustly myelinated in the third or fourth decade of life. A question therefore arises, as to whether changes in myelin observed during learning are a product of developmental myelination or a result of myelin plasticity of the adult CNS.

There is a large body of evidence indicating that myelin changes in the mature nervous system constitute a genuine example of experience-driven CNS plasticity. Social interaction, motor and cognitive training and changes in the environment can drive alterations in OPC number, oligodendrocyte production, myelin gene expression and myelin thickness in the adult CNS (Blumenfeld-Katzir et al., 2011; Liu et al., 2012; McKenzie et al., 2014; Okuda et al., 2009; Sampaio-Baptista et al., 2013; Simon et al., 2011; Xiao et al., 2016). In addition, it is clear that myelination of some white matter tracts is regulated by neuronal activity, suggesting a cellular mechanism by which experience can effect changes in myelin (Demerens et al., 1996; Gibson et al., 2014; Gyllensten and Malmfors, 1963).

On the other hand, myelination can be highly stereotypical, with little or no variation from individual to individual in certain CNS regions. In fact, myelination is unaffected following deprivation of sensory stimuli (Colello and Pott, 1997; Fukui et al., 1991; Etxeberria et al., 2016), under some circumstances, or intrinsic to certain brain areas (Bechler et al., 2015), and oligodendrocytes can differentiate in culture in the absence of neurons and myelinate silenced or paraformaldehyde-fixed axons and even engineered nanofibers (Bechler et al., 2015; Hines et al., 2015; Lee et al., 2012; Rosenberg et al., 2008). These observations indicate that, in some tracts, myelination is not driven by experience, but rather results from an innate program of development. Indeed, both activity-dependent and independent myelination have been observed in vivo (Koudelka et al., 2016) and in vitro (Lundgaard et al., 2013) where growth factors, such as neuroregulin-1 or BDNF, mediate a switch between the two modes of myelination, by upregulating NMDA receptors in oligodendrocyte linage cells making them more sensitive to glutamate released from active neurons (Lundgaard et al., 2013; Figure 3). Remarkably, social isolation and sensory deprivation in young mice impairs myelination in the relevant brain areas in a non-reversible manner and only during a critical period early in the postnatal life, suggesting that in other tracts, myelination may be better categorized as a form of developmental plasticity, reminiscent of experience-shaped development of neural circuit connections (Makinodan et al., 2012; Barreras et al., 2013). 
Thus, whether myelin changes reflect a developmental program of myelination or experiencedriven plasticity, largely depends on the fibre tract in question. It is possible that early myelination of tracts that are essential for survival and which show minimal individual variability - the first stage of myelination proposed by Flechsig (see the main text) - are bonafide examples of developmental myelination, much as early stages of CNS formation reflect an innate program of development. Myelination of other tracts during childhood - Flechsig's intermediate stage - may be sensitive to experience only during a limited time window, whereas myelination at later stages, that run far into adulthood, might be driven mainly by experience and represent a plasticity mechanism that circuits use to optimize their function accordingly to the environment. Myelin changes at these tracts correspond to the late stage of myelination as proposed by Flechsig and are reminiscent of synaptic plasticity mechanisms, which only continue to exist in the adult CNS in specific areas, such as the hippocampus.

\section{Cellular and molecular mechanisms underlying neuronal activity-regulated myelin plasticity}

A plethora of signals are known to regulate myelination, but the fact that myelination correlates with learning, and learning is driven by alterations in neuronal activity, makes it conceivable that neuronal activity-dependent myelination may be a driver for myelin plasticity. A number of mechanisms may in turn underlie regulation of myelination by neuronal activity.

\section{Axon-OPC synapses}

OPCs express neurotransmitter receptors, such as ionotropic glutamate receptors, and receive synaptic inputs from unmyelinated axons (or from unmyelinated segments of partially myelinated axons), making them able to sense and respond to changes in neuronal activity (Fannon et al., 2015; Gallo et al., 1996; Karadottir et al., 2005; Karadottir et al., 2008; Spampinato et al., 2014; Spitzer et al., 2016; Yuan et al., 1998). Thus, synaptic communication between unmyelinated axons and OPCs might underlie learning-dependent myelination. Glutamatergic inputs may be particularly important in this context, as over 90 percent of projection axons and association fibres are glutamatergic.

Axon-OPC synapses are found during development and throughout the mature CNS, in both grey and white matter (Bergles et al., 2000; Chittajallu et al., 2004; Gautier et al., 2015; Karadottir et al., 2005; Karadottir et al., 2008; Kukley et al., 2007; Lin and Bergles, 2004; 
Muller et al., 2009; Ziskin et al., 2007). Remarkably, overlapping populations of OPCs and postsynaptic neurons exhibit synchronized spontaneous activity, indicating that OPCs are indeed able to sense the pattern of activity arising in circuits (Mangin et al., 2008; Muller et al., 2009). Thus, axon-OPC synapses provide a cellular mechanism through which OPCs and the oligodendrocytes they generate can affect changes in myelin in response to neuronal activity. Evidence supporting a function of axon-OPC synapses and glutamate signalling in myelin plasticity comes from studies showing that block of vesicular release of glutamate or glutamate receptor activity impairs myelination (Lundgaard et al., 2013; Mensch et al., 2015; Wake et al., 2011). In addition, axon-OPC synapses are upregulated following demyelination and block of vesicular release, neuronal activity or glutamate receptors in demyelinated lesions prevents OPC differentiation and subsequent remyelination (Gautier et al., 2015). Collectively, these studies indicate that glutamate signalling via axon-OPC synapses is an important mechanism engaged by neuronal activity in the control of myelin plasticity.

\section{Activity dependent release of growth factors}

Neurotransmitters and growth factors are released in an activity-dependent manner and some are known to play a role in myelination. In particular, Neuregulin 1 (NRG1) and BrainDerived Neurotrophic Factor (BDNF) are critical to render myelination responsive to glutamate and neuronal activity (Lundgaard et al., 2013: see Figure 3). In the PNS, levels of NRG1 expression on the axonal surface correlates with myelination, and ectopic expression of NRG1 results in myelination of axons which otherwise would remain unmyelinated (Taveggia et al., 2005). This observation suggests that, in the developing PNS, NRG1 signalling through ErbB3 receptors provides a molecular mechanism for axon targeting by myelinating Schwann cells. Initial characterization of NRG1 function in the CNS revealed that no major alterations in myelin levels result from the absence of NRG1/ErbB3 signalling in oligodendrocytes (Brinkmann et al., 2008). However, further analysis on CNS specific areas indicated that thinner myelin profiles develop when ErbB3 is ablated in oligodendrocytes at a later time point of development (Makinodan et al., 2012). Thus, perhaps indicating that the different waves or cycles of myelination mentioned above may have a different molecular signal.

When soluble NRG1 or BDNF is added to dorsal root ganglion (DRG) neuron-OPC cocultures they induce a switch in myelination from an activity-independent to an activitydependent mode (Lundgaard et al., 2013). NRG1 and BDNF modulate the subunit composition of glial N-methyl-D-Aspartate receptors (NMDAR) to potentiate glutamateevoked, NMDAR-dependent OPC currents, rendering OPCs responsive to axon-derived glutamate and myelination sensitive to neuronal activity (Figure 3). These results suggest that 
NRG1 and BDNF signalling underlie myelination dependence on neuronal activity and provide mechanisms of axon target selection in the context of CNS myelin plasticity, where active axons are predominantly myelinated. Importantly, NRG1 expression itself is regulated by experience and neuronal activity, suggesting that as circuits mature and become active, NRG1 levels are up regulated and myelination switches to the activity-dependent mode (Makinodan et al., 2012; Ozaki et al., 2004).

Regulation of myelin plasticity by growth factors is reminiscent of synaptic plasticity as NRG1 also regulates the balance between excitatory and inhibitory neuronal transmission, synaptic plasticity and glutamate receptor density at the synapse depends on precisely regulated levels of NRG1 expression (Agarwal et al., 2014; Jiang et al., 2013; Li et al., 2007). Moreover, regulated BDNF secretion modulates both synaptic transmission and plasticity in the mature CNS (Figurov et al., 1996; Ji et al., 2010; Kang and Schuman, 1995). Thus, a commonality between myelin and synaptic plasticity emerges, in that activity-dependent secretion of growth factors, in particular NRG1 and BDNF, comprises a general mechanism employed by neuronal activity in the regulation of these two forms of plasticity in the adult CNS.

\section{PLEASE INSERT FIGURE 3 HERE}

\section{Axonal surface proteins}

However, synaptic input, along with growth factor signalling, is not likely to be the only mechanism regulating myelin plasticity, particularly because CNS axons develop individual myelin profiles, which are not necessarily uninterrupted along the axonal length (Deschenes and Landry, 1980; Remahl and Hildebrand, 1982; Tomassy et al., 2014). Regulated expression of axonal surface proteins is another mechanism that may underlie activityinduced changes in myelin; for example, the cell adhesion molecule L1. Low frequency $(0.1 \mathrm{~Hz})$ stimulation of DRG neurons in vitro down-regulates levels of L1, indicating that specific patterns of neuronal activity controls L1 expression in neurons (Itoh et al., 1995). Notably, addition of L1 blocking antibodies or L1-FC fusion proteins to myelinating cocultures impairs initiation of myelination, suggesting that neuron-glia interactions mediated by L1 are necessary for myelination induction (Barbin et al., 2004; Seilheimer et al., 1989; Wood et al., 1990) and that neuronal activity might control L1 expression as a mechanism for regulation of myelination. This has been indirectly demonstrated in vitro for the PNS, as lowfrequency stimulation impairs myelination and this is prevented by artificially raising L1 expression levels (Stevens et al., 1998). However, further experiments will be necessary to demonstrate if a comparable mechanism is in place during myelin plasticity in the CNS. 


\section{Axon-myelin synapse}

In addition to OPCs, cytoplasmic compartments of mature myelin sheaths also express ionotropic glutamate receptors (Butt et al., 2014; Karadottir et al., 2005). NMDAR-dependent calcium waves are elicited in the inner tongue of the myelin sheath in response to chemical ischaemia in vitro and this mechanism might play a role in white matter glutamatergic toxicity (Micu et al., 2006). In the healthy CNS, axon-myelin communication might regulate trophic support to axons, as stimulation of NMDAR mobilizes glucose transporters to the myelin sheath and conditional deletion of oligodendrocyte NMDAR impairs axonal recovery following oxygen-glucose deprivation (Saab et al., 2016). Remarkably, electrical stimulation of optic nerve axons induces calcium changes in the myelin sheath, which are NMDARdependent and sensitive to block of glutamate vesicular release (Micu et al., 2016) and artificial stimulation of motor cortex activity results in increased thickness of mature myelin sheaths (Gibson et al., 2014). These findings suggest that, in addition to axon-OPC synapses, direct communications between axons and the myelin sheath might exist that underlie activity-dependent, glutamate-mediated changes in myelin, primarily in sheath thickness and internode length (see BOX 1: Different forms of myelin plasticity).

A molecular mechanism potentially important to this form of modification is the regulation of myelin growth by PI3K/Akt signalling. Constitutive activation of Akt in oligodendrocytes induces continued myelination throughout the mouse life span (Flores et al., 2008) and tamoxifen-inducible deletion of PTEN - a PI3K inhibitor - reinitiates myelin growth in adult animals (Goebbels et al., 2010). These findings indicate that PI3K/Akt signalling provides a temporal component to the regulation of myelin growth, with activation of PI3K/Akt dictating the time of myelination initiation.

Recently, high-pressure freezing electron microscopy (EM) allowed for the identification of cytoplasmic channels running through forming myelin sheaths. These channels, which are reminiscent of the PNS Schimidt-Lanterman incisures, contain vesicles that provide membrane components to the active myelin growth zone at the inner tongue (Snaidero et al., 2014). With time, cytoplasmic channels are closed and are no longer observed after myelin is fully compacted. However, experimental elevation of PIP3 levels and activation of Akt signalling in myelinating oligodendrocytes, reopens cytoplasmic channels and reinitiates myelin growth in adult mice (Snaidero et al., 2014), suggesting a cellular mechanism, also dependent on PI3K/Akt signalling, through which myelin thickness and internode length may change after development. In principle, reopening of myelin cytoplasmic channels could also function as a cellular mechanism for sheath retraction and reduction of myelin thickness, 
although other mechanisms must be additionally in place, since, overall, activation of PI3K/Akt pathway results in hypermyelination (Flores et al., 2008; Goebbels et al., 2010). It has yet to be demonstrated if neuronal activity can regulate PI3K/Akt signalling to reactivate myelin growth. Intriguingly, NRG1, the expression of which is driven by experience (Makinodan et al., 2012; Ozaki et al., 2004), induces PI3K/Akt activation, and the increased myelination observed following NRG1 treatment of myelinating co-cultures in vitro is mediated via PI3K/Akt signalling (Lundgaard et al., 2013). Furthermore, activation of OPC glutamate receptors induces Akt signalling (Gallo et al., 1994), suggesting that activitydependent release of glutamate onto axon-OPC synapses or NRG1 secretion could be involved in re-initiation of myelin growth through PI3K/Akt signalling.

\section{Perspectives and conclusion}

Post-mortem anatomical studies implied more than a hundred years ago that myelination is sensitive to function of neuronal tracts and experience, and is able to adjust to changes in the environment (Bürgel et al., 2006; Olson et al., 2015). A significant amount of data has now accumulated indicating that changes in white matter correlate with learning and that myelination is adjusted by experience. It is now also recognized that neuronal activity can regulate myelination, and that this may be a mechanism by which experience effects changes in myelin. In this review, we have revisited the evidence supporting myelin plasticity and discussed the cellular and molecular mechanisms that may underlie neuronal activity-induced myelination in the adult CNS.

The acquisition of several cognitive and motor behaviours coincides with changes in myelin profiles in the developing and adult CNS. However, when reflecting on the role myelin plasticity plays during learning and memory, one should consider that changes occurring with learning are not unique to myelin and that experience also drives direct modifications on neuronal function - e.g. neurogenesis, synapse formation/elimination and changes in synapse strength. Thus, a fundamental open question in the field is whether myelin plasticity drives learning or if changes in myelin are rather a consequence of the modification of neuronal circuits. Remarkably, block of novel myelin formation in the adult CNS impairs motor learning (McKenzie et al., 2014), indicating that, whether a causal mechanism or mere consequence, myelin plasticity is needed for the modification of CNS function. Establishing the nature of the relationship between myelin and neural plasticity will require the direct demonstration that experience-driven changes in myelin profiles modulate the activity of intact neural circuits in a way that optimizes circuit performance. The development of new tools - e.g. high pressure freezing EM and optogenetics/pharmacogenetics - in recent years has been key to experimentally approach the phenomenon of myelin plasticity and to directly 
address the hypothesis made more than 100 years ago. Continued refinement and advancement of techniques will be paramount to directly address myelin plasticity.

A function of myelin in learning and memory also raises the possibility that neurological disorders, traditionally associated with neuronal dysfunction, may in fact arise from the disruption of myelin plasticity. Indeed, white matter defects have been observed in a variety of neurological and psychiatric diseases, including autism, attention-deficit hyperactivity disorder, schizophrenia, chronic depression and Alzheimer's disease (Fields, 2008; Nave and Ehrenreich, 2014). Investigation of this previously unappreciated link may therefore provide new therapeutic targets or strategies for a range of conditions and disorders that are currently difficult to treat. We recently identified that neuronal activity regulates remyelination via axon-OPC glutamate signalling (Gautier et al., 2015). This finding suggests that drugs modulating glutamatergic signalling may potentially promote myelin regeneration in demyelinating diseases.

Changes in neuronal function and, especially in synaptic properties, have historically dominated the scientific thinking and research effort aimed at understanding CNS plasticity. The exciting new evidence, reviewed here, suggests that activity-dependent, glutamatemediated changes in myelin might also serve as a cellular mechanism underlying plasticity. Research in the years to come will elucidate the mechanisms regulating myelin changes in the adult CNS, address whether myelin plasticity is confined to only certain tracts in the CNS and help to uncover the potential benefits of functionally modulating the plastic glia both in health and disease.

\section{References}

Agarwal, A., Zhang, M., Trembak-Duff, I., Unterbarnscheidt, T., Radyushkin, K., Dibaj, P., Martins de Souza, D., Boretius, S., Brzozka, M.M., Steffens, H., et al. (2014). Dysregulated expression of neuregulin-1 by cortical pyramidal neurons disrupts synaptic plasticity. Cell Rep 8, 1130-1145.

Arancibia-Carcamo, I.L., Ford, M.C., Cossell, L., Ishida, K., Tohyama, K., and Attwell, D. (2017). Node of Ranvier length as a potential regulator of myelinated axon conduction speed. Elife 6.

Balkowiec, A., and Katz, D.M. (2002). Cellular mechanisms regulating activity-dependent release of native brain-derived neurotrophic factor from hippocampal neurons. J Neurosci 22, 10399-10407. 
Barbin, G., Aigrot, M.S., Charles, P., Foucher, A., Grumet, M., Schachner, M., Zalc, B., and Lubetzki, C. (2004). Axonal cell-adhesion molecule L1 in CNS myelination. Neuron Glia Biol 1, 65-72.

Barrera, K., Chu, P., Abramowitz, J., Steger, R., Ramos, R.L., and Brumberg, J.C. (2013). Organization of myelin in the mouse somatosensory barrel cortex and the effects of sensory deprivation. Dev Neurobiol 73, 297-314.

Barres, B.A., and Raff, M.C. (1993). Proliferation of oligodendrocyte precursor cells depends on electrical activity in axons. Nature $361,258-260$.

Bechler, M.E., Byrne, L., and ffrench-Constant, C. (2015). CNS Myelin Sheath Lengths Are an Intrinsic Property of Oligodendrocytes. Current Biology 25, 2411-2416.

Bengtsson, S.L., Nagy, Z., Skare, S., Forsman, L., Forssberg, H., and Ullen, F. (2005). Extensive piano practicing has regionally specific effects on white matter development. Nat Neurosci 8, 1148-1150.

Bergles, D.E., Roberts, J.D., Somogyi, P., and Jahr, C.E. (2000). Glutamatergic synapses on oligodendrocyte precursor cells in the hippocampus. Nature 405, 187-191.

Bergmann, O., and Frisen, J. (2013). Neuroscience. Why adults need new brain cells. Science 340, 695-696.

Blumenfeld-Katzir, T., Pasternak, O., Dagan, M., and Assaf, Y. (2011). Diffusion MRI of structural brain plasticity induced by a learning and memory task. PLoS One 6, e20678.

Brinkmann, B.G., Agarwal, A., Sereda, M.W., Garratt, A.N., Müller, T., Wende, H., Stassart, R.M., Nawaz, S., Humml, C., Velanac, V., et al. (2008). Neuregulin-1/ErbB Signaling Serves Distinct Functions in Myelination of the Peripheral and Central Nervous System. Neuron 59, 581-595.

Bürgel, U., Amunts, K., Hoemke, L., Mohlberg, H., Gilsbach, J.M., and Zilles, K. (2006). White matter fiber tracts of the human brain: three-dimensional mapping at microscopic resolution, topography and intersubject variability. Neuroimage 29, 1092-1105.

Butt, A.M., Fern, R.F., and Matute, C. (2014). Neurotransmitter signaling in white matter. Glia 62, 1762-1779.

Carreiras, M., Seghier, M.L., Baquero, S., Estevez, A., Lozano, A., Devlin, J.T., and Price, C.J. (2009). An anatomical signature for literacy. Nature 461, 983-986. 
Chittajallu, R., Aguirre, A., and Gallo, V. (2004). NG2-positive cells in the mouse white and grey matter display distinct physiological properties. J Physiol 561, 109-122.

Colello, R.J., Devey, L.R., Imperato, E., and Pott, U. (1995). The chronology of oligodendrocyte differentiation in the rat optic nerve: evidence for a signaling step initiating myelination in the CNS. The Journal of Neuroscience 15, 7665-7672.

Colello, R.J., and Pott, U. (1997). Signals that initiate myelination in the developing mammalian nervous system. Molecular Neurobiology 15, 83-100.

Czopka, T., Ffrench-Constant, C., and Lyons, D.A. (2013). Individual oligodendrocytes have only a few hours in which to generate new myelin sheaths in vivo. Dev Cell 25, 599-609.

Dawson, M.R., Polito, A., Levine, J.M., and Reynolds, R. (2003). NG2-expressing glial progenitor cells: an abundant and widespread population of cycling cells in the adult rat CNS. Mol Cell Neurosci 24, 476-488.

De Biase, L.M., Kang, S.H., Baxi, E.G., Fukaya, M., Pucak, M.L., Mishina, M., Calabresi, P.A., and Bergles, D.E. (2011). NMDA receptor signaling in oligodendrocyte progenitors is not required for oligodendrogenesis and myelination. J Neurosci 31, 12650-12662.

Demerens, C., Stankoff, B., Logak, M., Anglade, P., Allinquant, B., Couraud, F., Zalc, B., and Lubetzki, C. (1996). Induction of myelination in the central nervous system by electrical activity. Proceedings of the National Academy of Sciences 93, 9887-9892.

Deoni, S.C., Mercure, E., Blasi, A., Gasston, D., Thomson, A., Johnson, M., Williams, S.C., and Murphy, D.G. (2011). Mapping infant brain myelination with magnetic resonance imaging. J Neurosci 31, 784-791.

Deschenes, M., and Landry, P. (1980). Axonal branch diameter and spacing of nodes in the terminal arborization of identified thalamic and cortical neurons. Brain Res 191, 538-544.

Etxeberria, A., Hokanson, K.C., Dao, D.Q., Mayoral, S.R., Mei, F., Redmond, S.A., Ullian, E.M., and Chan, J.R. (2016). Dynamic Modulation of Myelination in Response to Visual Stimuli Alters Optic Nerve Conduction Velocity. J Neurosci 36, 6937-6948.

Fannon, J., Tarmier, W., and Fulton, D. (2015). Neuronal activity and AMPA-type glutamate receptor activation regulates the morphological development of oligodendrocyte precursor cells. Glia 63, 1021-1035.

Fields, R.D. (2008). White matter in learning, cognition and psychiatric disorders. Trends Neurosci 31, 361-370. 
Figurov, A., Pozzo-Miller, L.D., Olafsson, P., Wang, T., and Lu, B. (1996). Regulation of synaptic responses to high-frequency stimulation and LTP by neurotrophins in the hippocampus. Nature 381, 706-709.

Flechsig, P. (1901). Developmental (mye-logenetic) localisation of the cerebral cortex in the human subject. Lancet 2, 1027-1029.

Flechsig, P.E. (1920). Anatomie des menschlichen Gehirns und Rückenmarks auf myelogenetischer Grundlage (G. Thieme).

Flores, A., Narayanan, S., Morse, E., Shick, H., Yin, X., Kidd, G., Avila, R., Kirschner, D., and Macklin, W. (2008). Constitutively active Akt induces enhanced myelination in the CNS. The Journal of neuroscience : the official journal of the Society for Neuroscience 28, 7174 7183.

Ford, M.C., Alexandrova, O., Cossell, L., Stange-Marten, A., Sinclair, J., Kopp-Scheinpflug, C., Pecka, M., Attwell, D., and Grothe, B. (2015). Tuning of Ranvier node and internode properties in myelinated axons to adjust action potential timing. Nat Commun $6,8073$.

Fukui, Y., Hayasaka, S., Bedi, K.S., Ozaki, H.S., and Takeuchi, Y. (1991). Quantitative study of the development of the optic nerve in rats reared in the dark during early postnatal life. Journal of Anatomy 174, 37-47.

Gallo, V., Patneau, D.K., Mayer, M.L., and Vaccarino, F.M. (1994). Excitatory amino acid receptors in glial progenitor cells: molecular and functional properties. Glia 11, 94-101.

Gallo, V., Zhou, J.M., McBain, C.J., Wright, P., Knutson, P.L., and Armstrong, R.C. (1996). Oligodendrocyte progenitor cell proliferation and lineage progression are regulated by glutamate receptor-mediated K+ channel block. J Neurosci 16, 2659-2670.

Gautier, H.O., Evans, K.A., Volbracht, K., James, R., Sitnikov, S., Lundgaard, I., James, F., Lao-Peregrin, C., Reynolds, R., Franklin, R.J., et al. (2015). Neuronal activity regulates remyelination via glutamate signalling to oligodendrocyte progenitors. Nat Commun 6, 8518 .

Gibson, E.M., Purger, D., Mount, C.W., Goldstein, A.K., Lin, G.L., Wood, L.S., Inema, I., Miller, S.E., Bieri, G., Zuchero, J.B., et al. (2014). Neuronal Activity Promotes Oligodendrogenesis and Adaptive Myelination in the Mammalian Brain. Science 344, 1252304.

Gibson, K.R. (1970). Sequence of myelinization in the brain of Macaca mulatta. University of California; Berkeley. 
Goebbels, S., Oltrogge, J.H., Kemper, R., Heilmann, I., Bormuth, I., Wolfer, S., Wichert, S.P., Mobius, W., Liu, X., Lappe-Siefke, C., et al. (2010). Elevated Phosphatidylinositol 3,4,5-Trisphosphate in Glia Triggers Cell-Autonomous Membrane Wrapping and Myelination. Journal of Neuroscience 30, 8953-8964.

Gyllensten, L., and Malmfors, T. (1963). Myelinization of the optic nerve and its dependence on visual function--a quantitative investigation in mice. J Embryol Exp Morphol 11, 255-266.

Hamilton, N.B., Clarke, L.E., Arancibia-Carcamo, I.L., Kougioumtzidou, E., Matthey, M., Karadottir, R., Whiteley, L., Bergersen, L.H., Richardson, W.D., and Attwell, D. (2017). Endogenous GABA controls oligodendrocyte lineage cell number, myelination, and CNS internode length. Glia 65, 309-321.

Hildebrand, C., Remahl, S., Persson, H., and Bjartmar, C. (1993). Myelinated nerve fibres in the CNS. Prog Neurobiol 40, 319-384.

Hines, J.H., Ravanelli, A.M., Schwindt, R., Scott, E.K., and Appel, B. (2015). Neuronal activity biases axon selection for myelination in vivo. Nature Neuroscience 18, 683-689.

Hofstetter, S., Tavor, I., Tzur Moryosef, S., and Assaf, Y. (2013). Short-term learning induces white matter plasticity in the fornix. J Neurosci 33, 12844-12850.

Huff, T.B., Shi, Y., Sun, W., Wu, W., Shi, R., and Cheng, J.X. (2011). Real-time CARS imaging reveals a calpain-dependent pathway for paranodal myelin retraction during highfrequency stimulation. PLoS One 6, e17176.

Hughes, E.G., Kang, S.H., Fukaya, M., and Bergles, D.E. (2013). Oligodendrocyte progenitors balance growth with self-repulsion to achieve homeostasis in the adult brain. Nat Neurosci 16, 668-676.

Itoh, K., Stevens, B., Schachner, M., and Fields, R.D. (1995). Regulated expression of the neural cell adhesion molecule L1 by specific patterns of neural impulses. Science 270, 13691372 .

Ji, Y., Lu, Y., Yang, F., Shen, W., Tang, T.T., Feng, L., Duan, S., and Lu, B. (2010). Acute and gradual increases in BDNF concentration elicit distinct signaling and functions in neurons. Nat Neurosci 13, 302-309.

Jiang, L., Emmetsberger, J., Talmage, D.A., and Role, L.W. (2013). Type III neuregulin 1 is required for multiple forms of excitatory synaptic plasticity of mouse cortico-amygdala circuits. J Neurosci 33, 9655-9666. 
Kaes, T. (1907). Die Grosshirnrinde des Menschen in ihren Massen und in ihrem Fasergehalt: Ein gehirnanatomischer Atlas mit erläuterndem Text und schematischer Zeichnung, 16 Tabellen, 15 Kurven und 79 farbigen Tafeln (G. Fischer).

Kang, H., and Schuman, E.M. (1995). Long-lasting neurotrophin-induced enhancement of synaptic transmission in the adult hippocampus. Science 267, 1658-1662.

Karadottir, R., Cavelier, P., Bergersen, L.H., and Attwell, D. (2005). NMDA receptors are expressed in oligodendrocytes and activated in ischaemia. Nature 438, 1162-1166.

Karadottir, R., Hamilton, N.B., Bakiri, Y., and Attwell, D. (2008). Spiking and nonspiking classes of oligodendrocyte precursor glia in CNS white matter. Nat Neurosci 11, 450-456.

Kinney, H.C., Brody, B.A., Kloman, A.S., and Gilles, F.H. (1988). Sequence of central nervous system myelination in human infancy. II. Patterns of myelination in autopsied infants. J Neuropathol Exp Neurol 47, 217-234.

Koudelka, S., Voas, M.G., Almeida, R.G., Baraban, M., Soetaert, J., Meyer, M.P., Talbot, W.S., and Lyons, D.A. (2016). Individual Neuronal Subtypes Exhibit Diversity in CNS Myelination Mediated by Synaptic Vesicle Release. Curr Biol 26, 1447-1455.

Kougioumtzidou, E., Shimizu, T., Hamilton, N.B., Tohyama, K., Sprengel, R., Monyer, H.Attwell, D., and Richardson, W.D. (2017). Signalling through AMPA receptors on oligodendrocyte precursors promotes myelination by enhancing oligodendrocyte survival. Elife 6.

Kukley, M., Capetillo-Zarate, E., and Dietrich, D. (2007). Vesicular glutamate release from axons in white matter. Nat Neurosci 10, 311-320.

Lakhani, B., Borich, M.R., Jackson, J.N., Wadden, K.P., Peters, S., Villamayor, A., MacKay, A.L., Vavasour, I.M., Rauscher, A., and Boyd, L.A. (2016). Motor Skill Acquisition Promotes Human Brain Myelin Plasticity. Neural Plast 2016, 7526135.

Lang, E.J., and Rosenbluth, J. (2003). Role of myelination in the development of a uniform olivocerebellar conduction time. J Neurophysiol 89, 2259-2270.

Lee, S., Chong, S.Y.C., Tuck, S.J., Corey, J.M., and Chan, J.R. (2013). A rapid and reproducible assay for modeling myelination by oligodendrocytes using engineered nanofibers. Nature Protocols 8, 771-782. 
Lee, S., Leach, M.K., Redmond, S.A., Chong, S.Y.C., Mellon, S.H., Tuck, S.J., Feng, Z.-Q., Corey, J.M., and Chan, J.R. (2012). A culture system to study oligodendrocyte myelination processes using engineered nanofibers. Nature Methods 9, 917-922.

Li, B., Woo, R.S., Mei, L., and Malinow, R. (2007). The neuregulin-1 receptor erbB4 controls glutamatergic synapse maturation and plasticity. Neuron 54, 583-597.

Li, Q., Brus-Ramer, M., Martin, J.H., and McDonald, J.W. (2010). Electrical stimulation of the medullary pyramid promotes proliferation and differentiation of oligodendrocyte progenitor cells in the corticospinal tract of the adult rat. Neurosci Lett 479, 128-133.

Lin, S.C., and Bergles, D.E. (2004). Synaptic signaling between GABAergic interneurons and oligodendrocyte precursor cells in the hippocampus. Nat Neurosci 7, 24-32.

Liu, J., Dietz, K., DeLoyht, J.M., Pedre, X., Kelkar, D., Kaur, J., Vialou, V., Lobo, M.K., Dietz, D.M., Nestler, E.J., et al. (2012). Impaired adult myelination in the prefrontal cortex of socially isolated mice. Nature Neuroscience 15, 1621-1623.

Long, P., and Corfas, G. (2014). Dynamic regulation of myelination in health and disease. JAMA Psychiatry 71, 1296-1297.

Lundgaard, I., Luzhynskaya, A., Stockley, J.H., Wang, Z., Evans, K.A., Swire, M., Volbracht, K., Gautier, H.O., Franklin, R.J., Charles, F.-C., et al. (2013). Neuregulin and BDNF induce a switch to NMDA receptor-dependent myelination by oligodendrocytes. PLoS Biol 11, e1001743.

Makinodan, M., Rosen, K.M., Ito, S., and Corfas, G. (2012). A critical period for social experience-dependent oligodendrocyte maturation and myelination. Science 337, 1357-1360.

Mangin, J.M., Kunze, A., Chittajallu, R., and Gallo, V. (2008). Satellite NG2 progenitor cells share common glutamatergic inputs with associated interneurons in the mouse dentate gyrus. J Neurosci 28, 7610-7623.

Matthews, M.A., and Duncan, D. (1971). A quantitative study of morphological changes accompanying the initiation and progress of myelin production in the dorsal funiculus of the rat spinal cord. J Comp Neurol 142, 1-22.

McKenzie, I.A., Ohayon, D., Li, H., de Faria, J.P., Emery, B., Tohyama, K., and Richardson, W.D. (2014). Motor skill learning requires active central myelination. Science 346, 318-322. 
Mensch, S., Baraban, M., Almeida, R., Czopka, T., Ausborn, J., El Manira, A., and Lyons, D.A. (2015). Synaptic vesicle release regulates myelin sheath number of individual oligodendrocytes in vivo. Nature Neuroscience 18, 628-630.

Micu, I., Jiang, Q., Coderre, E., Ridsdale, A., Zhang, L., Woulfe, J., Yin, X., Trapp, B.D., McRory, J.E., Rehak, R., et al. (2006). NMDA receptors mediate calcium accumulation in myelin during chemical ischaemia. Nature 439, 988-992.

Micu, I., Plemel, J.R., Lachance, C., Proft, J., Jansen, A.J., Cummins, K., van Minnen, J., and Stys, P.K. (2016). The molecular physiology of the axo-myelinic synapse. Experimental Neurology 276, 41-50.

Miller, D.J., Duka, T., Stimpson, C.D., Schapiro, S.J., Baze, W.B., McArthur, M.J., Fobbs, A.J., Sousa, A.M., Sestan, N., Wildman, D.E., et al. (2012). Prolonged myelination in human neocortical evolution. Proc Natl Acad Sci U S A 109, 16480-16485.

Muller, J., Reyes-Haro, D., Pivneva, T., Nolte, C., Schaette, R., Lubke, J., and Kettenmann, H. (2009). The principal neurons of the medial nucleus of the trapezoid body and NG2(+) glial cells receive coordinated excitatory synaptic input. J Gen Physiol 134, 115-127.

Nave, K.A., and Ehrenreich, H. (2014). Myelination and oligodendrocyte functions in psychiatric diseases. JAMA Psychiatry 71, 582-584.

Okuda, H., Tatsumi, K., Makinodan, M., Yamauchi, T., Kishimoto, T., and Wanaka, A. (2009). Environmental enrichment stimulates progenitor cell proliferation in the amygdala. J Neurosci Res 87, 3546-3553.

Olson, I.R., Von Der Heide, R.J., Alm, K.H., and Vyas, G. (2015). Development of the uncinate fasciculus: Implications for theory and developmental disorders. Dev Cogn Neurosci $14,50-61$.

Ozaki, M., Itoh, K., Miyakawa, Y., Kishida, H., and Hashikawa, T. (2004). Protein processing and releases of neuregulin-1 are regulated in an activity-dependent manner. Journal of Neurochemistry 91, 176-188.

Pajevic, S., Basser, P.J., and Fields, R.D. (2014). Role of myelin plasticity in oscillations and synchrony of neuronal activity. Neuroscience 276, 135-147.

Remahl, S., and Hildebrand, C. (1982). Changing relation between onset of myelination and axon diameter range in developing feline white matter. J Neurol Sci 54, 33-45. 
Reyners, H., Gianfelici de Reyners, E., Regniers, L., and Maisin, J.R. (1986). A glial progenitor cell in the cerebral cortex of the adult rat. J Neurocytol 15, 53-61.

Rivers, L.E., Young, K.M., Rizzi, M., Jamen, F., Psachoulia, K., Wade, A., Kessaris, N., and Richardson, W.D. (2008). PDGFRA/NG2 glia generate myelinating oligodendrocytes and piriform projection neurons in adult mice. Nat Neurosci 11, 1392-1401.

Rosenberg, S.S., Kelland, E.E., Tokar, E., Asia, R., and Chan, J.R. (2008). The geometric and spatial constraints of the microenvironment induce oligodendrocyte differentiation. Proceedings of the National Academy of Sciences 105, 14662-14667.

Saab, A.S., Tzvetavona, I.D., Trevisiol, A., Baltan, S., Dibaj, P., Kusch, K., Mobius, W., Goetze, B., Jahn, H.M., Huang, W., et al. (2016). Oligodendroglial NMDA Receptors Regulate Glucose Import and Axonal Energy Metabolism. Neuron 91, 119-132.

Salami, M., Itami, C., Tsumoto, T., and Kimura, F. (2003). Change of conduction velocity by regional myelination yields constant latency irrespective of distance between thalamus and cortex. Proc Natl Acad Sci U S A 100, 6174-6179.

Sampaio-Baptista, C., Khrapitchev, A.A., Foxley, S., Schlagheck, T., Scholz, J., Jbabdi, S., DeLuca, G.C., Miller, K.L., Taylor, A., Thomas, N., et al. (2013). Motor skill learning induces changes in white matter microstructure and myelination. The Journal of neuroscience: the official journal of the Society for Neuroscience 33, 19499-19503.

Scholz, J., Klein, M.C., Behrens, T.E., and Johansen-Berg, H. (2009). Training induces changes in white-matter architecture. Nat Neurosci 12, 1370-1371.

Seidl, A.H., and Rubel, E.W. (2016). Systematic and differential myelination of axon collaterals in the mammalian auditory brainstem. Glia 64, 487-494.

Seidl, A.H., Rubel, E.W., and Barria, A. (2014). Differential conduction velocity regulation in ipsilateral and contralateral collaterals innervating brainstem coincidence detector neurons. $\mathrm{J}$ Neurosci 34, 4914-4919.

Seilheimer, B., Persohn, E., and Schachner, M. (1989). Antibodies to the L1 adhesion molecule inhibit Schwann cell ensheathment of neurons in vitro. J Cell Biol 109, 3095-3103.

Shrager, P., and Novakovic, S.D. (1995). Control of myelination, axonal growth, and synapse formation in spinal cord explants by ion channels and electrical activity. Brain Res Dev Brain Res $88,68-78$. 
Simon, C., Gotz, M., and Dimou, L. (2011). Progenitors in the adult cerebral cortex: cell cycle properties and regulation by physiological stimuli and injury. Glia 59, 869-881.

Snaidero, N., Möbius, W., Czopka, T., Hekking, Liesbeth H.P., Mathisen, C., Verkleij, D., Goebbels, S., Edgar, J., Merkler, D., Lyons, David A., et al. (2014). Myelin Membrane Wrapping of CNS Axons by PI(3,4,5)P3-Dependent Polarized Growth at the Inner Tongue. Cell 156, 277-290.

Spampinato, S.F., Merlo, S., Chisari, M., Nicoletti, F., and Sortino, M.A. (2014). Glial metabotropic glutamate receptor-4 increases maturation and survival of oligodendrocytes. Front Cell Neurosci 8, 462.

Spitzer, S., Volbracht, K., Lundgaard, I., and Karadottir, R.T. (2016). Glutamate signalling: A multifaceted modulator of oligodendrocyte lineage cells in health and disease. Neuropharmacology 110, 574-585.

Stevens, B., Tanner, S., and Fields, R.D. (1998). Control of myelination by specific patterns of neural impulses. J Neurosci 18, 9303-9311.

Sturrock, R.R. (1980). Myelination of the mouse corpus callosum. Neuropathol Appl Neurobiol 6, 415-420.

Südhof, Thomas C. (2013). Neurotransmitter Release: The Last Millisecond in the Life of a Synaptic Vesicle. Neuron 80, 675-690.

Takeuchi, H., Taki, Y., Sassa, Y., Hashizume, H., Sekiguchi, A., Fukushima, A., and Kawashima, R. (2010). White matter structures associated with creativity: evidence from diffusion tensor imaging. Neuroimage 51, 11-18.

Tauber, H., Waehneldt, T.V., and Neuhoff, V. (1980). Myelination in rabbit optic nerves is accelerated by artificial eye opening. Neurosci Lett 16, 235-238.

Taveggia, C., Zanazzi, G., Petrylak, A., Yano, H., Rosenbluth, J., Einheber, S., Xu, X., Esper, R.M., Loeb, J.A., Shrager, P., et al. (2005). Neuregulin-1 type III determines the ensheathment fate of axons. Neuron 47, 681-694.

Tilney, F., Casamajor, L. (1924). Myelinogeny as applied to the study of behavior. Arch NeurPsych 12 (1), 1-66.

Tomassy, G.S., Berger, D.R., Chen, H.H., Kasthuri, N., Hayworth, K.J., Vercelli, A., Seung, H.S., Lichtman, J.W., and Arlotta, P. (2014). Distinct profiles of myelin distribution along single axons of pyramidal neurons in the neocortex. Science 344, 319-324. 
Trigo, D., and Smith, K.J. (2015). Axonal morphological changes following impulse activity in mouse peripheral nerve in vivo: the return pathway for sodium ions. J Physiol 593, 9871002.

Turrigiano, G. (2011). Too many cooks? Intrinsic and synaptic homeostatic mechanisms in cortical circuit refinement. Annu Rev Neurosci 34, 89-103.

Wake, H., Lee, P.R., and Fields, R.D. (2011). Control of local protein synthesis and initial events in myelination by action potentials. Science 333, 1647-1651.

Walhovd, K.B., Johansen-Berg, H., and Karadottir, R.T. (2014). Unraveling the secrets of white matter--bridging the gap between cellular, animal and human imaging studies. Neuroscience 276, 2-13.

Whitaker, K.J., Vertes, P.E., Romero-Garcia, R., Vasa, F., Moutoussis, M., Prabhu, G., Weiskopf, N., Callaghan, M.F., Wagstyl, K., Rittman, T., et al. (2016). Adolescence is associated with genomically patterned consolidation of the hubs of the human brain connectome. Proc Natl Acad Sci U S A 113, 9105-9110.

Wood, P.M., Schachner, M., and Bunge, R.P. (1990). Inhibition of Schwann cell myelination in vitro by antibody to the $\mathrm{L} 1$ adhesion molecule. J Neurosci 10, 3635-3645.

Xiao, L., Ohayon, D., McKenzie, I.A., Sinclair-Wilson, A., Wright, J.L., Fudge, A.D., Emery, B., Li, H., and Richardson, W.D. (2016). Rapid production of new oligodendrocytes is required in the earliest stages of motor-skill learning. Nat Neurosci 19, 1210-1217.

Xu, T., Yu, X., Perlik, A.J., Tobin, W.F., Zweig, J.A., Tennant, K., Jones, T., and Zuo, Y. (2009). Rapid formation and selective stabilization of synapses for enduring motor memories. Nature 462, 915-919.

Yakovlev, P.L., Lecours, A.R. (1967). The myelogenetic cycles of regional maturation of the brain, Vol 1 ( Oxford, UK: Blackwell Scientific).

Yeung, M.S., Zdunek, S., Bergmann, O., Bernard, S., Salehpour, M., Alkass, K., Perl, S., Tisdale, J., Possnert, G., Brundin, L., et al. (2014). Dynamics of oligodendrocyte generation and myelination in the human brain. Cell 159, 766-774.

Young, K.M., Psachoulia, K., Tripathi, R.B., Dunn, S.J., Cossell, L., Attwell, D., Tohyama, K., and Richardson, W.D. (2013). Oligodendrocyte dynamics in the healthy adult CNS: evidence for myelin remodeling. Neuron 77, 873-885. 
Yuan, X., Eisen, A.M., McBain, C.J., and Gallo, V. (1998). A role for glutamate and its receptors in the regulation of oligodendrocyte development in cerebellar tissue slices. Development 125, 2901-2914.

Zatorre, R.J., Fields, R.D., and Johansen-Berg, H. (2012). Plasticity in gray and white: neuroimaging changes in brain structure during learning. Nat Neurosci 15, 528-536.

Zhang, L.I., and Poo, M.M. (2001). Electrical activity and development of neural circuits. Nat Neurosci 4 Suppl, 1207-1214.

Zhang, J., Kolind, S.H., Laule, C., and MacKay, A.L. (2015). How does magnetization transfer influence mcDESPOT results? Magn Reson Med 74, 1327-1335.

Ziskin, J.L., Nishiyama, A., Rubio, M., Fukaya, M., and Bergles, D.E. (2007). Vesicular release of glutamate from unmyelinated axons in white matter. Nat Neurosci 10, 321-330. 


\section{Acknowledgement}

We would like to thank our funders: Lister Institute of Preventive Medicine: Ragnhildur Thóra Káradóttir; Allen Distinguished Investigator programme, through The Paul G. Allen Frontiers Group: Ragnhildur Thóra Káradóttir and Kimberley Evans; Biotechnology and Biological Sciences Research Council (BBSRC) and The Cambridge Trust: E. A. Claudia Pama; UK Multiple Sclerosis Society: Ragnhildur Thóra Káradóttir and Omar de-Faria-jr, Award number 50. The funders had no role in decision to publish, or preparation of the manuscript. 


\section{Figure legends}

\section{Figure 1 Development of human cortical myelination}

A schematic diagram of human cortical myelination, demonstrating how protracted cortical myelination is, and that does not reach its 'maximum' until very late in adult life. Numbers on the left indicate proximal location of the six cortical layers (I-VI). (Modified from Kaes histological drawings of human cortical myelination presented in his book published 1907, titled:"Die Grosshirnrinde des Menschen in ihren Massen und in ihrem Fasergehalt: Ein gehirnanatomischer Atlas mit erläuterndem Text und schematischer Zeichnung, 16 Tabellen, 15 Kurven und 79 farbigen Tafeln" edited and published by G. Fischer)

\section{Figure 2 Different forms of myelin plasticity}

Neuronal activity can in principle effect changes in myelin by four different mechanisms: (1) myelination of unmyelinated axons, (2) myelination of unmyelinated segments of partially myelinated axons, (3) structural modification of existing internodes and (4) architectural changes in the nodes of Ranvier.

\section{Figure 3 Two modes of myelination}

A schematic diagram of the two types of myelination programmes. (A) An activity independent mode of myelination and myelination that depends on neuronal activity. When the concentration of neuregulin is low, myelination occurs irrespective of neuronal activity. (B) When the concentration of neuregulin reaches a high level, it induces a switch to the activity-dependent myelination programme. Neuregulin signalling via ErbB receptors causes upregulation of NMDA receptor responses in oligodendrocytes, making them more sensitive to glutamate, released from active axons. This causes an increase in the myelination efficiency, it occurs faster and may allow oligodendrocytes to prioritise selection of firing axons over the inactive ones. (B') A diagram of identified intracellular signalling events underlying the activity-dependent myelination programme. Neuregulin binds to ErbB receptors on oligodendrocytes, activating the PI3K/Akt signalling cascade and inducing phosphorylation of the transcription factor Creb, which most likely regulates the expression of myelin genes. Neuregulin-ErbB signalling is potentiated by integrins. Calcium influx through NMDA receptors may enhance Akt and/or Creb signalling or increase myelination via a different signalling cascade. 